\title{
EFFECT OF PRESSURE FLUCTUATIONS ON THE TEMPERATURE DURING BRAKING
}

\author{
Katarzyna TOPCZEWSKA, Przemysław ZAMOJSKI \\ Faculty of Mechanical Engineering, Bialystok University of Technology, 45C Wiejska Str., 15-351 Bialystok, Poland \\ k.topczewska@pb.edu.pl, zamojski.przemyslaw@gmail.com
}

received 13 May 2020, revised 29 June 2020, accepted 1 July 2020

\begin{abstract}
The aim of this study is to develop the numerical-analytical model of frictional heating in a pad/disc system during braking including the pressure fluctuations, engendered by the pump in an anti-skid braking operation. For this purpose, the problem of motion and the one-dimensional thermal problem of friction for a semi-space/semi-space tribosystem were formulated and solved. Obtained solutions allow to calculate temperature distribution on the contact surface and inside the friction elements. Thermal analysis was performed for a metal-ceramic pad and a cast iron disc during one-time braking including the time-dependent, oscillating pressure. The influence of amplitude of pressure fluctuations on the temperature variations was investigated, especially on the value of maximum temperature achieved during braking.
\end{abstract}

Key words: Braking, Pressure fluctuations, Pad/disc system, Temperature

\section{INTRODUCTION}

Braking operation is performed by proper clamp of friction pads to the rotating disc. Therefore, the nominal value and variations of pressure determine the effectiveness of the friction process that occurs. In the case of severe, heavy-loaded braking processes, the spatial distribution of pressure on the contact surface can be assumed as uniform (Yevtushenko and Kuciej, 2012; Matysiak and Yevtushenko, 2001). However, the time profile of contact pressure can have various shapes, which has significant influence on the process.

Most known solutions for thermal friction problems have been obtained for a classic case of braking with constant deceleration, i.e. assuming that pressure is invariable in time (Fazekas, 1953; Belhocine and Bouchetara, 2012; Talati and Jalalifar, 2009; Nosko et al., 2012). More precise models use an exponential function to describe the increase of contact pressure at the beginning of braking to nominal value (Topczewska, 2018; Yevtushenko et al., 1999; Matysiak et al., 2002) or its simplified form - linear growth of pressure - and maintain the achieved nominal value to the end of braking (Topczewska, 2018; Yevtushenko and Grześ, 2015; Yevtushenko et al., 2019). In the article Topczewska (2018), the evolution of temperature on friction surface was found in a closed analytical form for a classic semi-space/semi-space system with regard to the time profiles of specific friction power corresponding to the exponential and linear increase of pressure. Another possible pressure variation during braking was taken into account indirectly by applying different profiles of specific friction power during single braking (Yevtushenko et al., 2019). In the abovementioned article, the analytical solutions of one-dimensional heat conduction problem formulated for semi-space/semi-space system were found.

All of the above-mentioned problems concerned processes with monotonically increasing pressure during braking. However, the growth of contact pressure may also be accompanied by its fluctuations caused by anti-slip regulation, anti-lock braking system or pulse braking mode. Thermal problems of friction contact pressure fluctuations during braking were formulated and solved for a strip/semi-space (Yevtushenko et al., 2010) and semispace/strip/semi-space tribosystems (Kuciej, 2011). In the latter articles, mainly, the influence of the time of pressure increase to nominal value and heat transfer through a contact surface at the temperature level were investigated.

In this paper, the distribution of temperature in the pad/disc system was found during braking with an exponential growth of pressure and its fluctuations. For this purpose, the initial problem of motion and the one-dimensional boundary value problem of heat conduction were considered. Performed numerical analysis allowed to establish the effect of amplitude of pressure oscillation on the contact surface during braking on the temperature distribution.

\section{STATEMENT OF THE PROBLEM}

One-time, rapid braking process of a pad/brake disc system was considered. The operating principle of this type of brake system is based on the friction pads' clamp on the surface of the rotating disc. As a result of friction, heat is generated on the contact surfaces. Investigations of temperature distribution in a heated friction element during braking at high sliding speed showed the following (Yevtushenko and Kuciej, 2012; Balakin and Sergienko, 1999):

1. Almost all frictional heat is absorbed by the pads and disc in an axial direction, i.e. perpendicular to the friction surface.

2. Significant gradients of temperature are present only at a short distance from the contact surface, the so-called effective depth of heat penetration (Chichinadze et al., 2010), which is much smaller than the actual thickness of friction elements.

3. The effect of convective heat exchange with the environment and wear are negligible (Yevtushenko et al., 2020; Topczewska et al., 2020). So, the whole energy generated during braking is converted into heat. 
4. Thermal resistance on the interface of pad and disc is inconsiderable. So, thermal contact is perfect.

In order to determine the temperature distribution in friction elements, proper initial problem of motion and thermal problem of friction were formulated on the basis of the above assumptions $(1-4)$.

\section{THE INITIAL PROBLEM OF MOTION}

The initial problem of motion for a pad/disc system during single braking from initial sliding velocity of the disc $V_{0}$ to the standstill position at $t_{s}$ has the following form (Kuciej, 2012):

$2 W_{0} V_{0}^{-2} \frac{d}{d t} V(t)=-f p(t) A_{k}, 0 \leq t \leq t_{s}$,

$V(0)=V_{0}$,

where $W_{0}$ is the initial kinetic energy, $f$ the coefficient of friction, $A_{k}=2 \pi\left(r_{e}^{2}-r_{i}^{2}\right)$ the nominal contact surface, $r_{e}, r_{i}$ the external and internal radii of a single friction surface and $p(t)$ is the contact pressure. Problem of motion (1), (2) can be written in the dimensionless form:

$\tau_{s}^{0} \frac{d}{d \tau} V^{*}(\tau)=-p^{*}(\tau), 0 \leq \tau \leq \tau_{s}$,

$V^{*}(0)=1$,

where the introduced dimensionless variables and parameters are:

$\tau=\frac{k_{1} t}{d^{2}}, \tau_{s}=\frac{k_{1} t_{s}}{d^{2}}, \tau_{s}^{0}=\frac{k_{1} t_{s}^{0}}{d^{2}}, t_{s}^{0}=\frac{2 W_{0}}{f V_{0} p_{0} A_{k}}$,

$V^{*}=\frac{V}{V_{0}}, p^{*}=\frac{p}{p_{0}}, d=\sqrt{3 k_{1} t_{s}^{0}}$,

where $p_{0}$ is the nominal value of pressure, $k_{1}$ the thermal diffusivity of the disc material and $d$ is the effective depth of heat penetration. Based on equation (3), taking into account of the initial condition (4), the dimensionless sliding velocity can be found using the equation:

$V^{*}(\tau)=1-\frac{1}{\tau_{s}^{0}} \int_{0}^{\tau} p^{*}(s) d s, 0 \leq \tau \leq \tau_{s}$.

Taking into account the growth of contact pressure at the beginning of braking, and also fluctuations of its value due to antilock braking system, the dimensionless time-dependent pressure function $p^{*}(\tau)$ is (Yevtushenko et al., 2010; Kuciej, 2011) calculated as:

$p^{*}(\tau)=\left[1-\exp \left(\frac{-\tau}{\tau_{m}}\right)\right][1+a \sin (\omega \tau)], 0 \leq \tau \leq \tau_{s}$,

where $t_{m}$ is the time of pressure increase, $\tau_{m}=k_{1} t_{m} d^{-2}$ the dimensionless time of pressure increase, $a$ the dimensionless amplitude of pressure oscillations and $\omega$ is the dimensionless frequency of pressure oscillations. With the analytical solution of equation (6) including pressure (7), the dimensionless sliding speed was found:

$$
\begin{gathered}
V^{*}(\tau)=1-\frac{\tau}{\tau_{s}^{0}}+\frac{\tau_{m}}{\tau_{s}^{0}}\left[1-\exp \left(\frac{-\tau}{\tau_{m}}\right)\right]-\frac{a}{\tau_{s}^{0}} V_{a}^{*}(\tau), 0 \leq \tau \leq \tau_{s}, \\
V_{a}^{*}(\tau)=\frac{1}{\omega}[1-\cos (\omega \tau)]+\frac{1}{\omega^{2}+\tau_{m}^{-2}}\left\{\exp \left(\frac{-\tau}{\tau_{m}}\right)[\omega \cos (\omega \tau)+\right. \\
\left.\left.+\tau_{m}^{-1} \sin (\omega \tau)\right]\right\}-\omega .
\end{gathered}
$$

The stop condition $V^{*}\left(\tau_{s}\right)=0$ allows to calculate numerically the time of braking.
For constant friction coefficient $f$, the specific friction power is the product of sliding velocity (8) and pressure (7):

$$
\begin{aligned}
q(t)= & q_{0} q^{*}(\tau), q_{0}=f p_{0} V_{0}, \\
q^{*}(\tau)= & {\left[1-\exp \left(\frac{-\tau}{\tau_{m}}\right)\right][1+a \sin (\omega \tau)]\left\langle 1-\frac{\tau}{\tau_{s}^{0}}+\frac{\tau_{m}}{\tau_{s}^{0}}[1-\right.} \\
& \left.\left.-\exp \left(\frac{-\tau}{\tau_{m}}\right)\right]-\frac{a}{\tau_{s}^{0}} V_{a}^{*}(\tau)\right\rangle, 0 \leq \tau \leq \tau_{s} .
\end{aligned}
$$
forms:

Substituting $a=0$, the obtained relations (7)-(9) take the

$$
\begin{aligned}
& p^{*}(\tau)=1-\exp \left(\frac{-\tau}{\tau_{m}}\right), V^{*}(\tau)=1-\frac{\tau}{\tau_{s}^{0}}+\frac{\tau_{m}}{\tau_{s}^{0}}\left[1-\exp \left(\frac{-\tau}{\tau_{m}}\right)\right], \\
& q^{*}(\tau)=\left[1-\exp \left(\frac{-\tau}{\tau_{m}}\right)\right]\left\langle 1-\frac{\tau}{\tau_{s}^{0}}+\frac{\tau_{m}}{\tau_{s}^{0}}\left[1-\exp \left(\frac{-\tau}{\tau_{m}}\right)\right]\right\rangle, \\
& 0 \leq \tau \leq \tau_{s},
\end{aligned}
$$

which correspond to braking with monotonically increasing pressure without fluctuations.

As the time of pressure growth approaches zero, $\tau_{m} \rightarrow 0$, from equations (7) to (10), the following equations were obtained:

$p^{*}(\tau)=1+a \sin (\omega \tau)$,

$V^{*}(\tau)=1-\frac{\tau}{\tau_{s}^{0}}-\frac{a}{\tau_{s}^{0}}\left\{\frac{1}{\omega}[1-\cos (\omega \tau)]-\omega\right\}$,

$q^{*}(\tau)=[1+a \sin (\omega \tau)]\left\langle 1-\frac{\tau}{\tau_{s}^{0}}-\frac{a}{\tau_{s}^{0}}\left\{\frac{1}{\omega}[1-\cos (\omega \tau)]-\omega\right\}\right\rangle$,

$0 \leq \tau \leq \tau_{s}$

And the simpliest typical braking process with uniform pressure can be found for $a=0$ and $\tau_{m} \rightarrow 0$ :

$$
\begin{aligned}
& p^{*}(\tau)=1, V^{*}(\tau)=1-\frac{\tau}{\tau_{s}^{0}}, q^{*}(\tau)=1-\frac{\tau}{\tau_{s}^{0}}, \\
& 0 \leq \tau \leq \tau_{s}=\tau_{s}^{0} .
\end{aligned}
$$

\section{THE HEAT PROBLEM OF FRICTION}

To establish the temperature field $T_{i}(z, t)$ in the brake disc ( $i=1$ ) and pad $(i=2)$, the thermal problem of friction was considered. Due to the symmetry of the system, we assume that the friction processes occurring on both friction surfaces of the brake disc are the same; so, the model was prepared for half of this system. Based on assumptions (1-4), half of the disc and the brake pad was simplified by a system of two semi-limited bodies. The orientation of semi-space/semi-space system considered here is given relative to a Cartesian frame of reference $O x y z$ (Fig. 1).

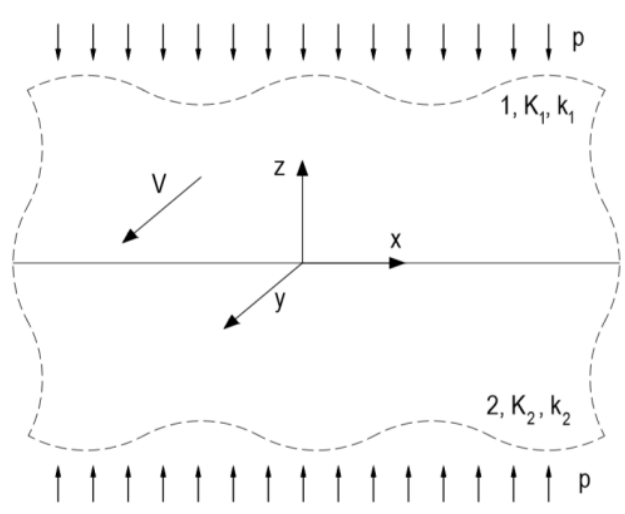

Fig. 1. Scheme of the problem 
On the contact surface, $z=0$; the frictional heat is generated and absorbed by elements along the $z$-axis direction. It was assumed that the friction materials are homogeneous and their thermal properties do not change during braking.

The boundary value problem of heat conduction was formulated for a semi-space/semi-space system in the dimensionless form:

$$
\begin{aligned}
& \frac{\partial^{2}}{\partial \zeta^{2}} T_{1}^{*}(\zeta, \tau)=\frac{\partial}{\partial \tau} T_{1}^{*}(\zeta, \tau), \zeta>0,0<\tau \leq \tau_{s}, \\
& \frac{\partial^{2}}{\partial \zeta^{2}} T_{2}^{*}(\zeta, \tau)=\frac{1}{k^{*}} \frac{\partial}{\partial \tau} T_{2}^{*}(\zeta, \tau), \zeta<0,0<\tau \leq \tau_{s} \\
& \left.K^{*} \frac{\partial}{\partial \zeta} T_{2}^{*}(\zeta, \tau)\right|_{\zeta=0}-\left.\frac{\partial}{\partial \zeta} T_{1}^{*}(\zeta, \tau)\right|_{\zeta=0}=q^{*}(\tau), 0<\tau \leq \tau_{s}, \\
& T_{1}^{*}(0, \tau)=T_{2}^{*}(0, \tau)=T^{*}(\tau), 0<\tau \leq \tau_{s} \\
& T_{i}^{*}(\zeta, \tau) \rightarrow 0,|\zeta| \rightarrow \infty, 0<\tau \leq \tau_{s}, i=1,2 \\
& T_{i}^{*}(\zeta, 0)=0,|\zeta| \geq 0, i=1,2,(18) \\
& \zeta=\frac{z}{d}, K^{*}=\frac{K_{2}}{K_{1}}, k^{*}=\frac{k_{2}}{k_{1}}, T_{0}=\frac{q_{0} d}{K_{1}}, \tau=\frac{k_{1} t}{d^{2}}, \tau_{s}=\frac{k_{1} t_{s}}{d^{2}}, \\
& T_{i}^{*}=\frac{T_{i}-T_{a}}{T_{0}}, d=\sqrt{3 k_{1} t_{s}^{0}}, i=1,2,
\end{aligned}
$$

where $T_{a}$ is the initial temperature.

Solution to the problem (13)-(19) was found based on the following Duhamel's theorem (Ozisik, 1993).

$$
\begin{aligned}
& T_{i}^{*}(\zeta, \tau)=\int_{0}^{\tau} q^{*}(s) \frac{\partial}{\partial \tau} T_{i}^{*(0)}(\zeta, \tau-s) d s, \\
& |\zeta| \geq 0,0 \leq \tau \leq \tau_{s}, i=1,2,
\end{aligned}
$$

where (Carlslaw and Jaeger, 1959)

$T_{i}^{*(0)}(\zeta, \tau)=2 \gamma \sqrt{\tau} \operatorname{ierfc}\left[Z_{i}(\zeta, \tau)\right], \zeta \geq 0,0 \leq \tau \leq \tau_{s}$

$Z_{1}(\zeta, \tau)=\frac{\zeta}{2 \sqrt{\tau}}, \zeta \geq 0, Z_{2}(\zeta, \tau)=\frac{\zeta}{2 \sqrt{\tau k^{*}}}, \zeta \leq 0$,

$\gamma=(1+\varepsilon)^{-1}, \varepsilon=K^{*}\left(k^{*}\right)^{-0.5}$

is the solution to the problem (13)-(19) with a constant intensity of frictional heat flux $q^{*}(\tau)=1$ in the boundary condition (15).

Taking into account the partial derivative of function (21) (Abramowitz and Stegun, 1972)

$\frac{\partial}{\partial \tau} T_{i}^{*(0)}(\zeta, \tau)=\gamma[\pi(\tau-s)]^{-0.5} \exp \left[-Z_{i}^{2}(\zeta, \tau-s)\right]$

and the obtained specific friction power $q^{*}(\tau)(9)$, we determined the temperature fields for the braking process considered here:

$T_{i}^{*}(\zeta, \tau)=\gamma \pi^{-0.5} \int_{0}^{\tau}\left[1-\exp \left(\frac{-s}{\tau_{m}}\right)\right][1+a \sin (\omega \tau)]\left\langle 1-\frac{s}{\tau_{s}^{0}}+\right.$

$+\frac{\tau_{m}}{\tau_{s}^{0}}\left[1-\exp \left(\frac{-s}{\tau_{m}}\right)\right]-$

$-\frac{a}{\tau_{s}^{0}}\left\{\frac{1}{\omega}[1-\cos (\omega s)]+\frac{1}{\omega^{2}+\tau_{m}^{-2}}\left\{\exp \left(\frac{-s}{\tau_{m}}\right)[\omega \cos (\omega s)+\right.\right.$

$$
\left.\left.\left.\left.+\tau_{m}^{-1} \sin (\omega s)\right]\right\}-\omega\right\}\right\rangle(\tau-s)^{-0.5} \exp \left[-Z_{i}^{2}(\zeta, \tau-s)\right] d s,
$$

$0 \leq \tau \leq \tau_{s},-\infty<\zeta<\infty, i=1,2$.

In the case of braking, when the time of pressure increase is close to zero, $\tau_{m} \rightarrow 0(11)$, the solution to the thermal friction problem (13)-(19) has the following form:

$T_{i}^{*}(\zeta, \tau)=\frac{\gamma}{\sqrt{\pi}} \int_{0}^{\tau}[1+a \sin (\omega \tau)]\left\langle 1-\frac{\tau}{\tau_{s}^{0}}-\frac{a}{\tau_{s}^{0}}\left\{\frac{1}{\omega}[1-\cos (\omega s)]-\right.\right.$ $-\omega\}\rangle(\tau-s)^{-0.5} \exp \left[-Z_{i}^{2}(\zeta, \tau-s)\right] d s$,

$0 \leq \tau \leq \tau_{s},-\infty<\zeta<\infty, i=1,2$.
Analytical integration of the formulas (23) and (24) is impossible. Therefore, it was done by means of the adaptive quadrature integrator, which handles singularities - procedure QAGS from a package of numerical integration QUADPACK (Piessens et al., 1983).

The analytical solution to the considered problem (13)-(19) without taking into account the pressure oscillations for $a=0$ has been succesfully found in the paper Topczewska (2018). Using the same method (20)-(22) for functions (10), the following formula has been obtained:

$$
\begin{gathered}
T^{*}(\tau)=\gamma \sqrt{\tau}\left[\left(1+\frac{\tau_{i}}{2 \tau_{s}^{0}}-\frac{2}{3} \frac{\tau}{\tau_{s}^{0}}\right) \frac{2}{\sqrt{\pi}}-\left(1-\frac{\tau}{\tau_{s}^{0}}+\frac{3}{2} \frac{\tau_{i}}{\tau_{s}^{0}}\right) D\left(\sqrt{\frac{\tau}{\tau_{i}}}\right)+\right. \\
\left.+\frac{\tau_{i}}{\tau_{s}^{0}} D\left(\sqrt{\frac{2 \tau}{\tau_{i}}}\right)\right], 0<\tau \leq \tau_{s}
\end{gathered}
$$

where

$D(x)=\frac{2}{\sqrt{\pi}} \frac{\exp \left(-x^{2}\right)}{x} \int_{0}^{x} \exp \left(s^{2}\right) d s$.

However, this solution, (25) and (26), allows to establish temperature evolution only of the friction surface $\zeta=0$, where the temperature level is the highest.

Also, the full analytical solution of this problem (13)-(19) has been determined for the uniform braking mode in the paper Yevtushenko et al. (2019):

$$
\begin{gathered}
T_{i}^{*}(\zeta, \tau)=\frac{4}{3} \gamma \sqrt{\tau} \frac{\tau}{\tau_{s}}\langle \\
\left\langle\left\{3 \frac{\tau_{s}}{\tau}-2\left[1+Z_{i}^{2}(\zeta, \tau)\right]\right\} \operatorname{ierfc}\left[Z_{i}(\zeta, \tau)\right]+\right. \\
\left.+Z_{i}(\zeta, \tau) \operatorname{erfc}\left[Z_{i}(\zeta, \tau)\right]\right\rangle,
\end{gathered}
$$

$|\zeta| \geq 0,0 \leq \tau \leq \tau_{s}, i=1,2$,

which allows to calculate temperature both on the contact surface and at any depth $|\zeta| \geq 0$ inside the friction elements.

\section{NUMERICAL ANALYSIS}

Distribution of temperature during braking was designated for the friction pair consisting of the pad made of metal-ceramic composite FMC-11 and the brake disc made of cast iron ChNMKh. Properties of these materials and input parameters to perform analysis are presented in Table 1.

Table 1. Input parameters (Yevtushenko et al., 2020; Kuciej, 2012)

\begin{tabular}{|c|c|}
\hline Thermal conductivity of disc, $K_{1}(\mathrm{~W} / \mathrm{mK})$ & 51 \\
\hline Thermal conductivity of pad, $K_{2}(\mathrm{~W} / \mathrm{mK})$ & 34.3 \\
\hline Thermal diffusivity of disc, $k_{1}\left(\mathrm{~mm}^{2} / \mathrm{s}\right)$ & 14 \\
\hline Thermal diffusivity of pad, $k_{2}\left(\mathrm{~mm}^{2} / \mathrm{s}\right)$ & 15.2 \\
\hline Initial velocity, $V_{0}(\mathrm{~m} / \mathrm{s})$ & 23.8 \\
\hline Initial kinetic energy, $W_{0}(\mathrm{k})$ & 103.54 \\
\hline Nominal contact pressure, $p_{0}(\mathrm{MPa})$ & 0.607 \\
\hline Friction coefficient, $f(-)$ & 0.27 \\
\hline Time of pressure increase, $t_{m}(\mathrm{~s})$ & 0.5 \\
\hline Ambient temperature, $T_{a}\left({ }^{\circ} \mathrm{C}\right)$ & 20 \\
\hline External radius, $r_{e}(\mathrm{~mm})$ & 37.5 \\
\hline Internal radius, $r_{i}(\mathrm{~mm})$ & 26.5 \\
\hline Pressure fluctuation amplitude, $a(-)$ & 0.1 \\
\hline Pressure fluctuation frequency, $\omega(-)$ & 100 \\
\hline
\end{tabular}


Based on the values included in Table 1, the following parameters were computed: the time of braking $t_{s}=12.43 \mathrm{~s}$, the whole area of friction pair contact $A_{k}=4.42 \times 10^{-3} \mathrm{~m}^{2}$, effective depth of heat penetration in the brake disc $d=22.45 \mathrm{~mm}$ and the coefficient of heat flux partition $\gamma=0.607$.

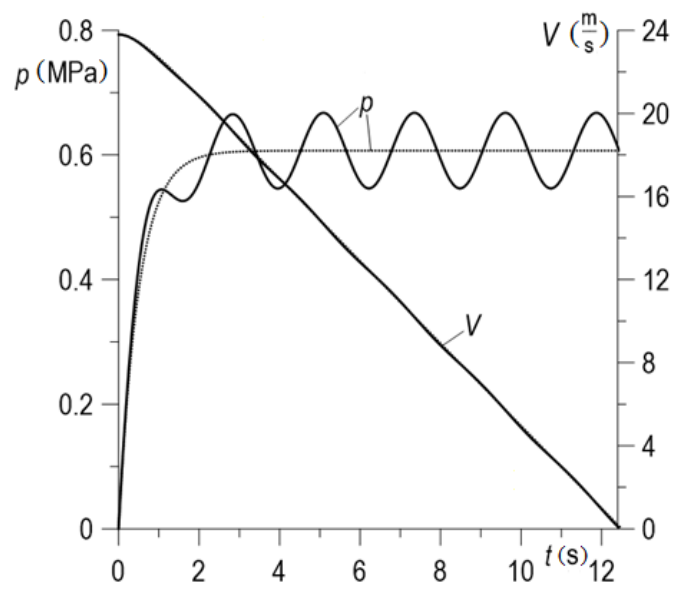

Fig. 2. Changes of contact pressure $p$ and sliding speed $V$ during braking on account of pressure fluctuations for $a=0.1$ (solid lines), (7), (8) and without for $a=0$ (dashed lines) (6), (10)

Changes in contact pressure $p$ and sliding speed $V$ during braking are shown in Fig. 2. Solid lines present the results obtained for oscillations with amplitude $a=0.1(7),(8)$, and dashed lines show the results obtained excluding the pressure fluctuations for $a=0$ (10). In the first case (for $a=0.1$ ), at the beginning of the process, the pressure rapidly increases. After that, it oscillates around the nominal value $p_{0}$ with a constant amplitude to the end of braking $t=t_{s}$. However, the sliding velocity monotonically decreases in time until the stop moment. The time profiles of speed in both cases are almost convergent (Fig. 2).

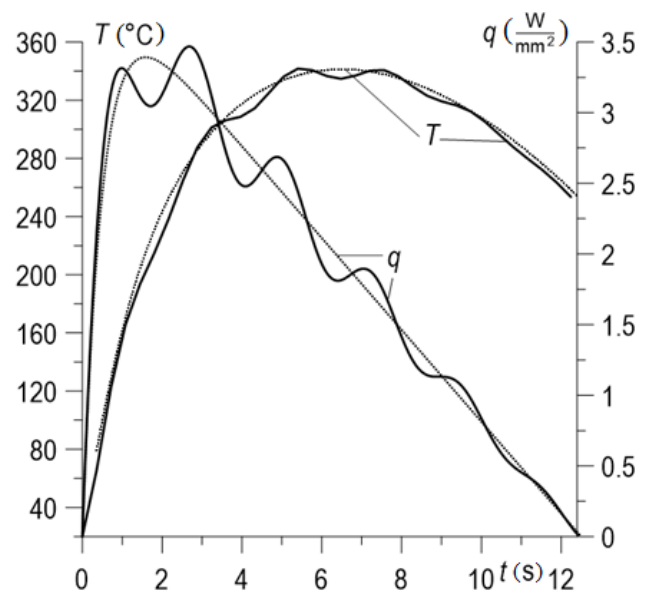

Fig. 3. Changes of specific friction power $q(9)$ and temperature $T$ on the contact surface during braking: including the pressure fluctuations for $a=0.1$ (solid lines) (23) and excluding for $a=0$ (dashed lines) (24)

Pressure oscillations with $a=0.1$ noticeably affect specific friction power $q(9)$ and temperature $T$ (23), (24) on the contact surface $z=0$, which is demonstrated in Fig. 3 by solid lines. The amplitude of their fluctuations is the greatest near the time moment of reaching their maximum values $q_{\max }=3.46 \mathrm{~W} / \mathrm{mm}^{2}$ and $T_{\max }=339.5^{\circ} \mathrm{C}$ at time moments $t=2.66 \mathrm{~s}$ and $t=6.64 \mathrm{~s}$, respectively, and after that it decreases until the end of braking. Temperature oscillations on the friction surface are much less intense than the fluctuations of specific friction power.

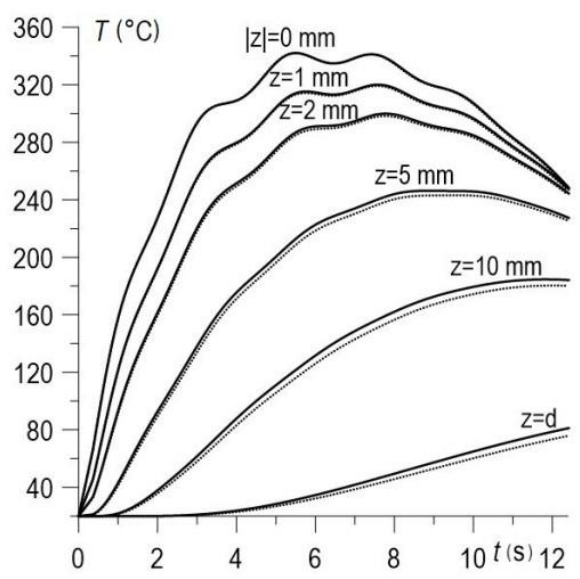

Fig. 4. Variations of temperature $T$ (23) on the contact surfaces $z=0$ and on selected distance from $z$ in the disc $(i=1)$ (solid lines) and pad $(i=2)$ (dotted lines)

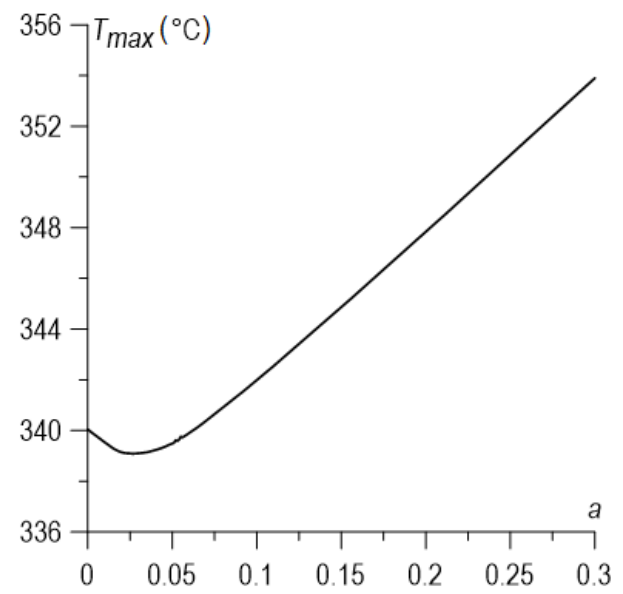

Fig. 5. Dependence of maximum temperature $T_{\max }$ attained during braking on the value of dimensionless amplitude $a$

Changes in temperature $T$ on the interface and few depths $Z$ inside the brake disc $(i=1)$ (solid lines) and pad $(i=2)$ (dotted lines) were calculated based on formula (23) and are presented in Fig. 4. The temperature values on the contact surfaces $|z|=0$ are the highest and the same for both friction pair elements (16). Due to similarity of thermal properties of friction materials (Table 1), the values of achieved temperature at selected distances in both elements are close to each other. The greater the distance from the friction surface $z$, the more noticeable are the differences between the temperature in the disc and the pad. As the distance from the friction surface increases, the temperature level decreases and its maximum values are reached later. The influence of pressure oscillations on temperature is especially visible on the friction surfaces $z=0$, and moving away from this surface, the fluctuation amplitude decreases. Below the depth $z=5 \mathrm{~mm}$, this effect is not noticeable both in the disc and the brake pad. 
Figure 5 presents the maximum temperature values achieved on the contact surface during braking for different values of fluctuations amplitude $a$. When braking without contact pressure oscillation $a=0$, the maximum temperature value is $T_{\max }=$ $340^{\circ} \mathrm{C}$. The lowest value of maximum temperature $T_{\max }=339^{\circ} \mathrm{C}$ is attained for small pressure fluctuations with an amplitude $a=0.03$. However, further increasing the amplitude value causes increase in maximum temperature to $T_{\max }=353.9^{\circ} \mathrm{C}$ at the most intense oscillations for an amplitude $a=0.3$.

\section{CONCLUSIONS}

Numerical analysis of temperature distribution in a pad/disc brake system was performed based on the solution to the boundary value problem of heat conduction formulated with oscillating pressure. Calculations were performed for the frictional pair such as composite pad and cast iron disc. Results were compared with data obtained excluding the pressure fluctuations. The following conclusions were made:

1. Pressure fluctuations do not influence the sliding speed of the brake disc, and hence the time of braking.

2. The temperature in the disc and pad oscillates only in the near distance from friction surface and with a much lower intensity than the corresponding fluctuations in pressure. The amplitude of temperature oscillations changes over time during braking and achieves the highest value in the middle of process, despite the fact that pressure fluctuates with a constant amplitude.

3. Increase in the level of amplitude of pressure fluctuations causes a slight increase in the value of maximum temperature attained on the friction surface.

\section{REFERENCES}

1. Abramowitz M., Stegun I.A. (1972), Handbook of Mathematical Functions with Formulas, Graphs and Tables, National Bureau of Standards, Washington.

2. Balakin V. A., Sergienko V. P. (1999), Thermal calculations of brakes and units of friction, IMMS NANB, Gomel.

3. Belhocine A., Bouchetara M. (2012), Thermal analysis of a solid brake disc. Appl. Therm. Eng., Vol. 32, 59-67.

4. Carlslaw H.S., Jaeger J. C. (1959), Conduction of Heat in Solids, 2nd ed.Clarendon Press, Oxford.

5. Chichinadze A.V., Kozhemyakina V.D., Suvorov A.V. (2010), Method of temperature-field calculation in model ring specimens during bilateral friction in multidisc aircraft brakes with the IM-58-T2 new multipurpose friction machine, J. Friction. Wear, Vol. 31, No. 1 23-32.

6. Fazekas G.A.G. (1953), Temperature gradients and heat stresses in brake drums, SAE Trans. Vol. 61, 279-284.

7. Kuciej M. (2011), Accounting changes of pressure in time in onedimensional modeling the process of friction heating of disc brake. Int. J. Heat Mass Transfer, 54 (1-3), 468-474.

8. Kuciej M. (2012), Analytical Models of Transient Friction Heating Oficyna Wydawnicza Politechniki Białostockiej, Białystok (in Polish).

9. Matysiak S. J., Yevtushenko A. A., Ivanyk E. G. (2002), Contact temperature and wear of composite friction elements during braking. Int. J. Heat Mass Transfer, Vol. 45, No. 1, 193-199.

10. Matysiak S.J., Yevtushenko A.A. (2001), On heating problems of friction, J. Theor. Appl. Mech., Vol. 39, No. 3, 577-588.
11. Nosko A.L., Mozalev V.V., Nosko A.P., Suvorov A.V., Lebedeva V.N. (2012), Calculation of temperature of carbon disks of aircraft brakes with account of heat exchange with the environment, J. Friction. Wear, Vol. 33, No. 4, 233-238.

12. Ozisik M.N. (1993), Heat conduction, 2nd Ed. Wiley: New York.

13. Piessens R., de Doncker-Kapenga E., Überhuber C.W., Kahaner D.K. (1983), QUADPACK: A Subroutine Package for Automatic Integration, Springer-Verlag, Berlin.

14. Talati F., Jalalifar S. (2009), Analysis of heat conduction in a disk brake system, Heat Mass Transfer, Vol. 45, 1047-1059.

15. Topczewska K. (2018), Influence of the Time of Increase in Contact Pressure in the Course of Braking on the Temperature of a Pad-Disc Tribosystem, Materials Science, Vol. 54, No. 2, 250-259.

16. Topczewska K., Schlattmann J., Abdullah O.I. (2020), Temperature and thermal stresses distributions in a dry friction clutch. J. Theor. Appl. Mech., Vol. 58, No. 2, 351-360.

17. Yevtushenko A., Grześ P. (2015), Maximum temperature in a threedisc thermally nonlinear braking system, Int. J. Heat Mass Transfer, Vol. 68, 291-298.

18. Yevtushenko A., Kuciej M. (2012), One-dimensional thermal problem of friction during braking: The history of development and actual state, Int. J. Heat Mass Transfer, Vol. 55, 4148-4153.

19. Yevtushenko A., Kuciej M., Topczewska K. (2019), Effect of the temporal profile of the friction power on temperature of a pad-disc brake system, J. Theor. Appl. Mech., Vol. 57, No. 2, 461-473.

20. Yevtushenko A., Kuciej M., Topczewska K. (2020), Some theoretical model for determining the temperature field of a multi-disk brake. Adv. Mech. Eng., Vol. 12, No. 1, 1-15.

21. Yevtushenko A.A., Ivanyk E.G., Yevtushenko 0.0. (1999), Exact formulae for determination of the mean temperature and wear during braking. Heat Mass Transfer, Vol. 35, No. 2, 163-169.

22. Yevtushenko A.A., Kuciej M., Yevtushenko O.O. (2010), Influence of the pressure fluctuations on the temperature in pad/disc tribosystem. Int. J. Heat Mass Transfer, Vol. 37, No. 8, 978-983. 